\title{
PREVALÊNCIA DE TRANSTORNOS MENTAIS COMUNS ENTRE ESTUDANTES UNIVERSITÁRIOS DA ÁREA DA SAÚDE: REVISÃO INTEGRATIVA
}

\section{PREVALENCE OF COMMON MENTAL DISORDERS AMONG UNIVERSITY STUDENTS IN THE HEALTH AREA: INTEGRATIVE REVIEW}

\author{
Maryelli Laynara Barbosa de Aquino Santos ${ }^{1}$ \\ Luana da Silva Leal ${ }^{2}$ \\ Raquel Pontes de Brito $^{3}$ \\ Luciana Karla Viana Barroso ${ }^{4}$
}

RESUMO: OBJETIVO: Este estudo tem por objetivo identificar os fatores que levam os estudantes universitários da área de saúde a desenvolverem TMCs e como esses transtornos interferem no desempenho acadêmico. METODOLOGIA: Trata-se de uma revisão integrativa da literatura e, para a elaboração deste estudo utilizou-se o portal PUBMED (Biblioteca Nacional de Medicina e Instituto Nacional de Saúde); bases de dados PEDRO (Physiotherapy Evidence Database) e SCIELO (Biblioteca Eletrônica Científica Online). Foram incluídos artigos brasileiros, originais envolvendo, os termos de pesquisa: Saúde Mental. Depressão. Ansiedade. Estudantes de Ciências da Saúde, publicados nos últimos quinze anos e excluídos os que abrangiam estudantes universitários de outras áreas e estudos realizados em outros países; os artigos encontrados em duplicidade foram contabilizados apenas uma vez. Após a aplicação dos critérios de inclusão e exclusão, apenas 12 artigos foram selecionados para análise. RESULTADOS: Foi encontrado que o surgimento dos TMCs está estritamente associado a rotinas de estudos excessivas, eventos de estresse, ausência de descanso e lazer, falta de apoio familiar e social. Causando impactos negativos em todos os segmentos da vida e suas relações sociais e pessoais. CONCLUSÃO: Os TMCs são desencadeados pelos seguintes fatores:

\footnotetext{
1 Graduanda. do curso de Fisioterapia do Centro Universitário - UNIFACISA, Campina Grande, Paraíba. E-mail: maryelli.santos@maisunifacisa.com.br.

2 Graduanda. do curso de Fisioterapia do Centro Universitário - UNIFACISA, Campina Grande, Paraíba.

${ }^{3}$ Graduanda. do curso de Fisioterapia do Centro Universitário - UNIFACISA, Campina Grande, Paraíba.

${ }^{4}$ Docente de Saúde da Criança e Anatomia do Centro Universitário - UNIFACISA, Campina Grande, Paraíba. E-mail: Ikarlab@yahoo.com.br.
} 
rotina de estudos excessiva, estresse, ausência de descanso e lazer, falta de apoio familiar e social afetando o desempenho acadêmico, a produtividade, a qualidade de vida e o bem-estar psicossocial, causando sofrimento mental e dificuldade de socialização dentro e fora da universidade. Este estudo instiga os estudantes e a instituição acadêmica ao desenvolvimento de ações preventivas e ao autocuidado.

Palavras chave: Ansiedade. Depressão. Estudantes de Ciências da Saúde. Saúde Mental.

ABSTRACT: OBJECTIVE: This study aims to identify the factors that lead university students in the health field to develop CMDs and how these disorders interfere with academic performance. METHODOLOGY: This is an integrative literature review and, for the preparation of this study, the portal PUBMED (National Library of Medicine and National Institute of Health) was used; PEDRO (Physiotherapy Evidence Database) and SCIELO (Online Electronic Scientific Library) databases. Brazilian articles, originals involving the search terms: Mental Health, were included. Depression. Anxiety. Health Sciences students, published in the last fifteen years and excluding those that included university students from other areas and studies carried out in other countries; duplicate articles were counted only once. After applying the inclusion and exclusion criteria, only 12 articles were selected for analysis. RESULTS: It was found that the appearance of CMDs is strictly associated with excessive study routines, stress events, lack of rest and leisure, lack of family and social support. Causing negative impacts in all segments of life and their social and personal relationships. CONCLUSION: CMDs are triggered by the following factors: excessive study routine, stress, lack of rest and leisure, lack of family and social support affecting academic performance, productivity, quality of life and psychosocial well-being, causing suffering mental and socialization difficulties inside and outside the university. This study encourages students and the academic institution to develop preventive actions and self-care.

Keywords: Anxiety. Depression. Health Sciences students. Mental Health. 


\section{INTRODUÇÃO}

Os Transtornos Mentais Comuns (TMCs) são conceituados como transtornos mentais e/ou transtornos não psicóticos, os quais referem-se a quadros com menor gravidade e maior frequência (SILVA; COSTA, 2012; GRETHER et al., 2019). Estudos com parâmetros ampliados para TMCs evidenciam que aproximadamente $30 \%$ dos adultos brasileiros apresentam sintomas tais como: estados mistos de depressão e ansiedade, insônia, fadiga, irritabilidade, esquecimento, dificuldade de concentração e de tomada de decisões, além de queixas somáticas (cefaléia, falta de apetite, tremores, má digestão, e outros) (SCHMIDT et al., 2011; GRANER; CERQUEIRA, 2019).

Segundo Neponuceno, Carvalho e Neves (2019) o modelo atual de ensino afasta-se de aspectos psicológicos e aproxima-se da formação técnico-científica, supervalorizando a cobrança por práticas que potencializam o desempenho acadêmico, passando despercebido os elementos fundamentais para manutenção do equilíbrio entre mente e corpo. Logo, o estudante universitário, especialmente aqueles da área da saúde, precisam pesar na balança o custo-benefício entre estudar muito/abdicar da vida social versus conciliar a rotina exaustiva de estudos entre vida social e manutenção da qualidade de vida; o que pode ser visto pelos seus semelhantes como uma escolha que o tornará um profissional mediano ou inferior.

Aspectos vivenciados na formação acadêmica, tais como: pressão excessiva, elevado nível de exigência impostos por si mesmo e pela academia, a sobrecarga de disciplinas e conteúdos, o tempo reduzido para o lazer e cuidado pessoal, a competitividade entre colegas, o contato com doenças e a vivência de episódios de óbitos dos pacientes são identificados como eventos de impacto ou perturbação, e que podem trazer prejuízos importantes à saúde (LIMA et al., 2019). Somado a isso, a tentativa frustrada de conciliar tudo ao mesmo tempo é um dos fatores que leva ao desgaste mental desses jovens (NEPONUCENO; CARVALHO; NEVES, 2019). 
Além disso, o fato de ter que lidar com vidas após a formação, faz com que a academia exija do aluno certas características que trabalham o senso de responsabilidade, a capacidade de tomada de decisão em favor do paciente, ou o conhecimento total sobre questões complexas, sendo fatores de máximo estresse no âmbito acadêmico, portanto, a soma de situações crônicas de estresse tem levado ao crescente número de TMCs em acadêmicos nos últimos tempos (SERRA; DINATO; CASEIRO, 2015; LIMA et al., 2019).

Entende-se que, o acometimento da saúde mental exerce forte interferência sobre a vida acadêmica e social do estudante, contribuindopara uma baixa produtividade, engajamento, dificuldade na retenção de informações; além de dificultar os relacionamentos em todas as suas esferas, ocasionando desgaste e adoecimento psíquico, levando ao uso de fármacos controlados a fim de melhorar a performance, e consequente risco de suicídio (COSTA, et al., 2012; LEÃO et al., 2018). Logo, dados sobre TMCs são relevantes para contribuir para a criação de ações preventivas e de autocuidado responsável (FIOROTTI et al., 2010; GRETHER et al., 2019).

Deste modo, este estudo tem por objetivo identificar os fatores que levam os estudantes universitários da área de saúde a desenvolverem TMCs e como esses transtornos interferem diretamente no desempenho acadêmico.

\section{METODOLOGIA}

Trata-se de uma revisão integrativa da literatura, tendo utilizado: o portal PUBMED (Biblioteca Nacional de Medicina e Instituto Nacional de Saúde); e bases de dados PEDRO (Physiotherapy Evidence Database) e SCIELO (Biblioteca Eletrônica Científica Online). A estratégia de pesquisa envolveu os seguintes termos: "saúde mental, depressão, ansiedade, Estudantes de Ciências da Saúde", nos idiomas inglês e português e, destes, foram incluídos os artigos brasileiros, originais envolvendo os termos de pesquisa em estudantes universitários na área da saúde, publicados nos últimos quinze anos, e foram excluídos os que incluíam estudantes 
universitários de outras áreas e estudos realizados em outros países; os artigos encontrados em duplicidade foram contabilizados apenas uma vez. Após a aplicação dos critérios de inclusão e exclusão, apenas 12 artigos foram selecionados para análise.

\section{RESULTADOS}

Um resumo da busca eletrônica nas bases de dados selecionadas é apresentado na Figura 1, na qual observa-se que foram identificados 305 artigos, dos quais 208 foram excluídos por não possuírem dados relevantes ou apresentarse em duplicidade e, 76 foram excluídos por outros critérios. Desses, 21 foram lidos na íntegra, dos quais apenas 12 artigos preenchiam os critérios de inclusão, sendo assim, selecionados para esta revisão integrativa.

Figura 1: Estratégia de busca e seleção de artigos.

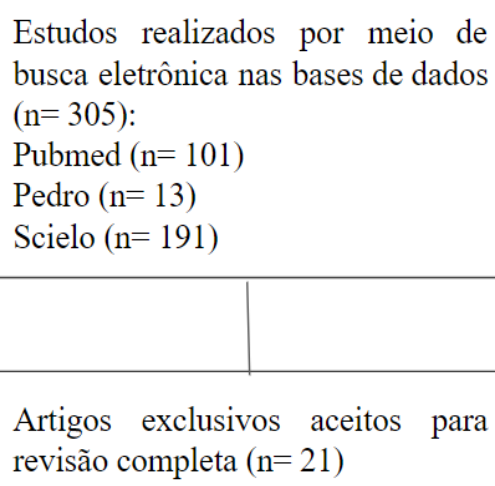

Total de artigos incluídos para análise $(\mathrm{n}=12)$

Fonte: Próprio Autor.
Excluídos na revisão abstrata $(\mathrm{n}=284)$

Estudos em duplicata $(\mathrm{n}=40)$

Estudos não relacionados ao tema $(\mathrm{n}=168)$

Critérios de exclusão $(n=76)$

- Estudantes universitários de outras áreas $(n=42)$

- Estudos realizados em outros países $(\mathrm{n}=$ 34) 
$\mathrm{Na}$ Tabela 1 são apresentados, resumidamente, os artigos que foram selecionados, o tamanho amostral, as característica metodológicas e os principais resultados encontrados em cada estudo.

Tabela 1: Estratificação dos estudos para análise por autor e ano, amostra, métodos e principais resultados.

\begin{tabular}{|c|c|c|c|}
\hline $\begin{array}{l}\text { ESTUDOS } \\
\text { (anos) }\end{array}$ & AMOSTRA & MÉTODOS & RESULTADOS \\
\hline $\begin{array}{l}\text { GRANER; } \\
\text { CERQUEIR } \\
\text { A (2019) }\end{array}$ & 37 ARTIGOS & $\begin{array}{l}\text { REVISÃO } \\
\text { LITERATURA }\end{array}$ & $\begin{array}{l}\text { OBSERVOU-SE A PREVALÊNCIA DE } \\
\text { SOFRIMENTO PSIQQUICO DE } 18,5 \% \\
\text { A 49,1\%, FATORES DE RISCO, } \\
\text { CONDIÇÕES RELATIVAS Á VIDA } \\
\text { ACADÊMICA E Â SAÚDE. NOS DOZE } \\
\text { ESTUDOS IDENTIFICARAM } \\
\text { FATORES DE PROTEÇÃO, SENSO } \\
\text { DE COERÊNCIA, AUTOEFICÁCIA, } \\
\text { VIGOR, AUTOESTIMA, RESILIÊNCIA, } \\
\text { ENTRE OUTRAS CONDIÇÕES } \\
\text { PSICOLÓGICAS. }\end{array}$ \\
\hline $\begin{array}{l}\text { GRETHER } \\
\text { et } \\
(2019)\end{array}$ & $\begin{array}{l}340 \text { ESTUDANTES } \\
\text { UNIVERSITÁRIOS } \\
\text { MATRICULADOS } \\
\text { NO CURSO DE } \\
\text { MEDICINA }\end{array}$ & $\begin{array}{l}\text { DOIS } \\
\text { QUESTIONÁRIOS } \\
\text { ANÔNIMOS DE } \\
\text { AUTO RESPOSTA, } \\
\text { UM PARA COLETA } \\
\text { DE DADOS } \\
\text { SOCIODEMOGRÁFI } \\
\text { COS E OUTRO } \\
\text { PARA } \\
\text { RASTREAMENTO } \\
\text { DE TRANSTORNOS } \\
\text { MENTAIS COMUNS } \\
\text { (TMC). }\end{array}$ & 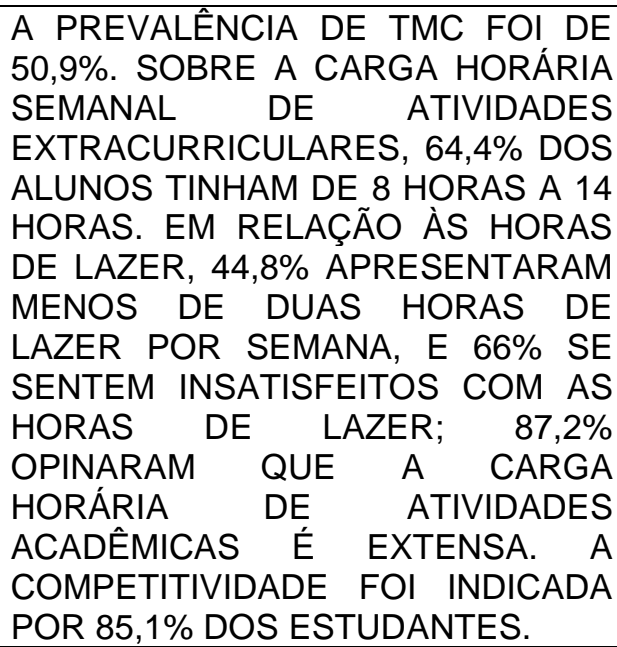 \\
\hline $\begin{array}{l}\text { LIMA et al. } \\
(2019)\end{array}$ & $\begin{array}{l}383 \text { ESTUDANTES } \\
\text { UNIVERSITÁRIOS }\end{array}$ & $\begin{array}{lr}\text { FOI UTILIZADA } & \text { A } \\
\text { APLICAČÃO } & \text { DO } \\
\text { INVENTÁRIO } & \text { DE } \\
\text { DEPRESSÃO } & \text { BECK } \\
\text { - BDI. } & \end{array}$ & $\begin{array}{l}\text { A PREVALÊNCIA DE DEPRESSÃO } \\
\text { ENTRE OS ACADÊMICOS FOI DE } \\
\text { 62,92\%. NO CURSO DE MEDICINA A } \\
\text { PREVALÊNCIA FOI DE 22,73\%, NO } \\
\text { CURSO DE ENFERMAGEM } \\
71,02 \% \text { E NO CURSO DE } \\
\text { ODONTOLOGIA 60,64\%. }\end{array}$ \\
\hline $\begin{array}{l}\text { SILVA; } \\
\text { COSTA } \\
(2012)\end{array}$ & $\begin{array}{l}455 \text { ESTUDANTES } \\
\text { UNIVERSITÁRIOS } \\
\text { DE AMBOS OS } \\
\text { SEXOS }\end{array}$ & $\begin{array}{l}\text { APLICAÇÃO DE UM } \\
\text { INSTRUMENTO DE } \\
\text { AUTO- } \\
\text { PREENCHIMENTO } \\
\text { SELF-REPORTING } \\
\text { QUESTIONNAIRE } \\
\text { (SRQ-20). }\end{array}$ & $\begin{array}{l}\text { NESSE ESTUDO A AMOSTRA DE } \\
\text { MAIOR PREVALÊNCIA SOBRE TMCS } \\
\text { SE MOSTROU MAIS RECORRENTE } \\
\text { EM MULHERES (88\%) DO CURSO } \\
\text { DE FISIOTERAPIA }(40 \%) .\end{array}$ \\
\hline $\begin{array}{l}\text { ESTUDOS } \\
\text { (anos) }\end{array}$ & AMOSTRA & MÉTODOS & RESULTADOS \\
\hline $\begin{array}{l}\text { SANTOS et } \\
\text { al (2017) }\end{array}$ & $\begin{array}{l}115 \text { ESTUDANTES } \\
\text { UNIVERSITÁRIOS }\end{array}$ & $\begin{array}{l}\text { A QUALIDADE DE } \\
\text { VIDA FOI AVALIADA }\end{array}$ & $\begin{array}{l}\text { A PREVALÊNCIA DE TMC FOI DE } \\
32,2 \% \text { O MENOR DOMÍNIO DA }\end{array}$ \\
\hline
\end{tabular}




\begin{tabular}{|c|c|c|c|}
\hline & $\begin{array}{l}\text { DE AMBOS OS } \\
\text { SEXOS }\end{array}$ & $\begin{array}{lr}\text { MEDIANTE } & \\
\text { APLICAÇÃO DO } \\
\text { WHOQOL-BREF. A } \\
\text { AVALIAÇÂO DE } \\
\text { TMC } & \text { FOI } \\
\text { MENSURADA POR } \\
\text { MEIO DO SELF } \\
\text { REPORTING } \\
\text { QUESTIONNAIRE } \\
\text { (SRQ-20). }\end{array}$ & $\begin{array}{l}\text { QUALIDADE DE VIDA ENTRE OS } \\
\text { INDIVÍDUOS COM TMC FOI O MEIO } \\
\text { AMBIENTE COM MEDIANA DE 56,4, } \\
\text { SEGUIDO PELO PSICOLÓGICO 56,9; } \\
\text { FÍSICO 57,1; E RELAÇÕES SOCIAIS } \\
65,5 .\end{array}$ \\
\hline $\begin{array}{l}\text { AUERBAC } \\
\mathrm{H} \text { et al. } \\
(2016)\end{array}$ & $\begin{array}{l}\text { ESTUDANTES } \\
\text { UNIVERSITÁRIOS } \\
(\mathrm{N}=1572) \text { E NÃO- } \\
\text { ESTUDANTES (N } \\
=\quad 4178) \text {, } \\
\text { INCLUINDO NÂO- } \\
\text { ESTUDANTES } \\
\text { QUE NÂO SE } \\
\text { FORMARAM POR } \\
\text { ABANDONO } \\
\text { DECURSO (N = } \\
\text { 702) DE AMBOS } \\
\text { OS SEXOS. }\end{array}$ & $\begin{array}{l}\text { OS INQUÉRITOS } \\
\text { MUNDIAIS } \quad \text { DE } \\
\text { SAÚDE MENTAL DA } \\
\text { ORGANIZAÇÃO } \\
\text { MUNDIAL DA } \\
\text { SAÚDE }\end{array}$ & $\begin{array}{l}\text { OS TRANSTORNOS DE ANSIEDADE } \\
\text { FORAM A CLASSE DE DISTÚRBIOS } \\
\text { MAIS PREVALENTE EM TODOS OS } \\
\text { GRUPOS DA AMOSTRA COMPLETA, } \\
\text { SEGUIDA PELOS TRANSTORNOS } \\
\text { DE HUMOR, TRANSTORNOS POR } \\
\text { SUBSTÂNCIAS E TRANSTORNOS } \\
\text { COMPORTAMENTAIS O OS } \\
\text { TRANSTORNOS INDIVIDUAIS MAIS } \\
\text { PREVALENTES FORAM FOBIAS E } \\
\text { TRANSTORNO DEPRESSIVO MAIOR } \\
\text { - TDM. }\end{array}$ \\
\hline $\begin{array}{l}\text { FACUNDE } \\
\text { S; } \\
\text { LUDERMIR } \\
(2005)\end{array}$ & $\begin{array}{l}443 \text { ESTUDANTES } \\
\text { UNIVERSITÁRIOS }\end{array}$ & $\begin{array}{l}\text { SELF REPORTING } \\
\text { QUESTIONNAIRE - } \\
\text { SRQ - } 20 \text { E UM } \\
\text { QUESTIONÁRIO } \\
\text { SOBRE } \\
\text { CARACTERÍSTICAS } \\
\text { DO PROCESSO } \\
\text { ENSINO- } \\
\text { APRENDIZAGEM }\end{array}$ & $\begin{array}{l}\text { A PREVALENCIA TOTAL DOS } \\
\text { TRANSTORNOS MENTAIS COMUNS } \\
\text { FOI DE } 34,1 \%, \quad \text { SENDO } \\
\text { SIGNIFICATIVAMENTE } \\
\text { ENTRE OS QUE SE SENTIAM } \\
\text { SOBRECARREGADOS E OS QUE } \\
\text { AFIRMARAM A PRESENÇA DE } \\
\text { SITUAÇÕES ESPECIAIS DURANTE } \\
\text { A INFÂNCIA E ADOLESCENCIA. }\end{array}$ \\
\hline $\begin{array}{l}\text { LEÃO et al } \\
(2018)\end{array}$ & $\begin{array}{l}476 \text { ESTUDANTES } \\
\text { UNIVERSITÁRIOS } \\
\text { COM } \\
\text { PREDOMÍNIO DO } \\
\text { SEXO FEMININO. }\end{array}$ & $\begin{array}{l}\text { FORAM } \\
\text { APLICADOS TRÊS } \\
\text { QUESTIONÁRIOS: } \\
\text { 1ASPECTOS } \\
\text { SOCIODEMOGRÁFI } \\
\text { COS; } \\
\text { 2OINVENTÁRIO DE } \\
\text { DEPRESSÃO BECK } \\
\text { (BDI) (ADAPTAÇÃO } \\
\text { E PADRONIZAÇÃO } \\
\text { BRASILEIRA); } \\
\text { 3oINVENTÁRIO DE } \\
\text { ANSIEDADE BECKK } \\
\text { (BAI) (ADAPTAÇÃO } \\
\text { E PADRONIZAÇÃO } \\
\text { BRASILEIRA) }\end{array}$ & $\begin{array}{l}\text { AS } \\
\text { PREVALÊNCIAS }\end{array}$ \\
\hline $\begin{array}{l}\text { NEPONUC } \\
\text { ENO; } \\
\text { CARVALH } \\
\text { O; NEVES } \\
(2019)\end{array}$ & $\begin{array}{l}16 \text { PUBLICAÇÕES } \\
\text { PERTINENTES AO } \\
\text { TEMA } \\
\text { INVESTIGADO. }\end{array}$ & $\begin{array}{l}\text { REVISÃO } \\
\text { LITERATURA }\end{array}$ & 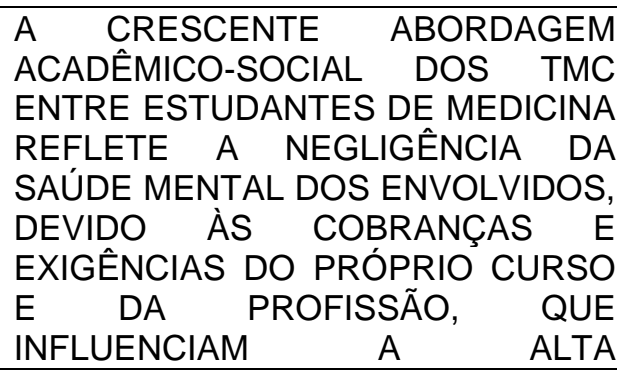 \\
\hline
\end{tabular}




\begin{tabular}{|c|c|c|c|}
\hline & & & $\begin{array}{lcr}\text { PREVALÊNCIA } & \text { DE } & \text { SUICÍDIO, } \\
\text { DEPRESSÃO E USO DE DROGAS. }\end{array}$ \\
\hline $\begin{array}{c}\text { ESTUDOS } \\
\text { (anos) }\end{array}$ & AMOSTRA & MÉTODOS & RESULTADOS \\
\hline $\begin{array}{l}\text { ARINÕ; } \\
\text { GABARDI } \\
\text { (2018) }\end{array}$ & $\begin{array}{l}640 \\
\text { GRADUANDOS }\end{array}$ & $\begin{array}{l}\text { O PROTOCOLO } \\
\text { DIGITAL } \\
\text { QUATRO } \\
\text { SESSÕES: } \\
\text { REGISTRO DO } \\
\text { TERMO } \\
\text { CONCORDÂNCIA, DE } \\
\text { QUESTIONÁRIO } \\
\text { SOCIODEMOGRÁFI } \\
\text { CO, DEPRESSION } \\
\text { ANXIETY STRESS } \\
\text { SCALE - DASS-21, } \\
\text { ESCALA } \\
\text { AUTOEFICÁCIA DE } \\
\text { PARA FORMAÇÃO } \\
\text { SUPERIOR - EAFS }\end{array}$ & \begin{tabular}{lcr} 
OS RESULTADOS & INDICAM \\
RELAÇÕES & SIGNIFICATIVAS, \\
NEGATIVAS, & \multicolumn{2}{c}{ ANSIEDADE, } \\
DEPRESSÃO E & STRESS COM A \\
AUTOEFICÁCIA & E COM COM A \\
QUALIDADE & DAS & VIVÊNCIAS \\
ACADÊMICAS. SOBRESSAINDO \\
QUADROS DE DEPRESSÃO SOBRE \\
OS DE ANSIEDADE.
\end{tabular} \\
\hline $\begin{array}{l}\text { COSTA et } \\
\text { al (2017) }\end{array}$ & $\begin{array}{l}5 \text { ARTIGOS E } 2 \\
\text { DISSERTAÇÕES }\end{array}$ & $\begin{array}{l}\text { REVISÃO } \\
\text { LITERATURA }\end{array}$ & 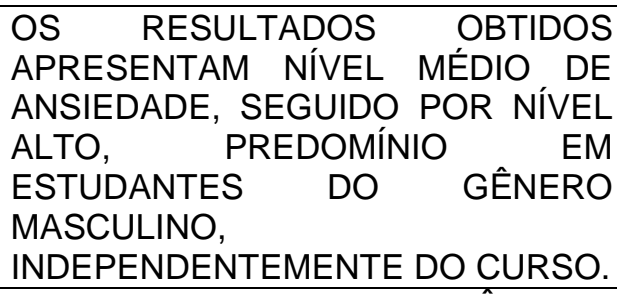 \\
\hline $\begin{array}{l}\text { ESTRELA } \\
\text { et al (2018) }\end{array}$ & $\begin{array}{ll}138 \text { ALUNOS } & \text { DO } \\
\text { CURSO } & \text { DE } \\
\text { MEDICINA } & \end{array}$ & 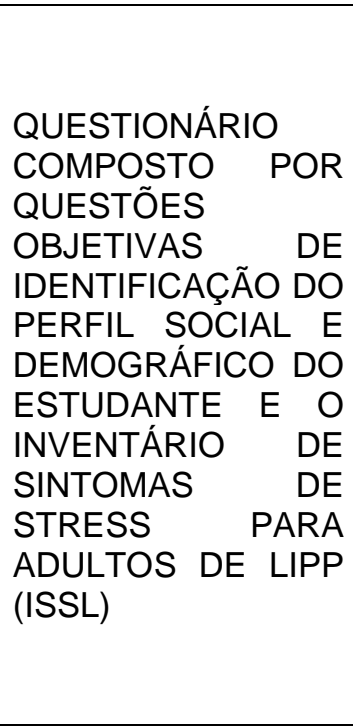 & 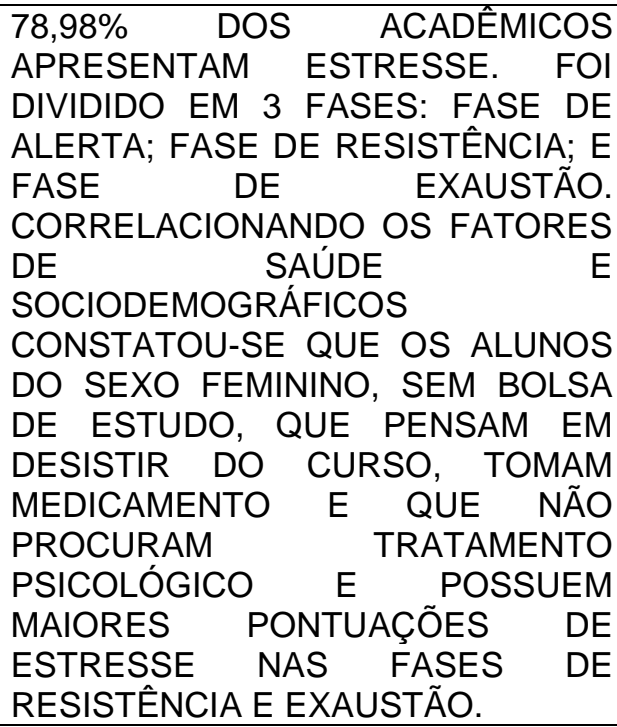 \\
\hline
\end{tabular}

Fonte: Próprio Autor.

Estudos apontam que há uma desarmonia entre o equilíbrio mental e corporal de universitários da área de saúde e que os transtornos mentais podem, além de afetar a vida, interferir no futuro dessas pessoas, cujos impactos não podem ser calculados ou mensurados. Logo, é importante compreendê-los no âmbito das relações humanas, a fim de elencar soluções que melhorem os aspectos 
relacionados à qualidade de vida de forma global desses estudantes (LIMA et al., 2019).

Silva e Costa (2015) analisaram que há uma maior predisposição para o acometimento de TMCs durante a fase de admissão acadêmica, do que na fase intermediária e final do curso, tendo estas últimas fases apresentado um percentual de $28 \%$ de acometimento. No estudo de Grether et al. (2019) a prevalência de TMCs na população estudada foi de 50,9\%, resultante de fatores acadêmicos e sociais; em que houve mais casos de TMCs entre: a) Estudantes que nunca pensaram em desistir do curso; b) Estudantes com atividades acadêmicas superiores a oito horas/dia; c) Estudantes com média de lazer inferior a duas horas/dia; somado a isso, senso de competitividade, problemas de socialização, sentimento de rejeição e ausência de apoio emocional.

Lima et al. (2019) em sua pesquisa, salienta que a prevalência de depressão nos acadêmicos da área de saúde foi significativa, sendo os maiores índices os sintomas depressivos, observados no curso de Enfermagem, atingindo prevalentemente o sexo feminino. Silva e Costa (2015) evidenciaram a prevalência de casos de depressão em $88 \%$ do sexo feminino, dessa vez, em estudantes do curso de Fisioterapia.

Santos et al. (2017) identificou em sua amostra que a prevalência de TMCs de $32,2 \%$, resultou em menor domínio na qualidade de vida, de meio ambiente, psicológico, físico e de relações sociais. Auerbach et al. (2016) percebeu na sua amostra que os transtornos de ansiedade foram a classe de distúrbios mais prevalentes, representando $28,6 \%$. Leão et al. (2018) constata as maiores prevalências em depressão $(28,6 \%)$ e ansiedade $(36,1 \%)$ nos estudantes que apresentaram insatisfação com seus respectivos cursos e que estes têm cerca de quatro vezes mais chances de desenvolver depressão. Quanto à prevalência de ansiedade, esta esteve mais associada ao sexo feminino e entre estudantes que apresentavam relacionamento insatisfatório entre familiares, amigos e colegas.

Neponuceno, Carvalho e Neves (2019) observaram em sua amostragem um crescente número de TMCs entre os estudantes de medicina, supondo que exista certa negligência quanto ao autocuidado e acerca da saúde mental destes. Segundo estes autores esse índice é resultado das excessivas cobranças e exigências 
inerentes ao curso, que por sua vez, influenciam diretamente em eventos de suicídio, depressão, uso de drogas, distúrbios conjugais e disfunções acadêmicas, refletindo em incapacidade temporária ou permanente de desempenhar seu papel acadêmico e profissional. Já Anacleto-Estrela et al. (2008) observou em sua pesquisa um elevado índice de estresse, sobretudo em estudantes do sexo feminino, sem bolsa de estudo, que pensam em desistir do curso, ou que tomam medicamentos e não procuram por tratamento psicológico especializado.

Costa et al. (2017) identificou um nível médio de chances para o desenvolvimento de TMCs entre os estudantes do sexo masculino, seguido por um nível alto de ansiedade, independentemente do curso. Por sua vez, Ariño e Bardagi (2018) tiveram, através dos seus resultados, a confirmação da sua hipótese geral, indicando relações significativas, negativas, como: ansiedade, depressão, stress com a autoeficácia e com a qualidade das vivências acadêmicas.

Facundes e Ludermir (2005) evidenciaram em seu estudo que a prevalência geral de TMCs ocorreu entre os estudantes de medicina com 42,6\%, em comparação com os estudantes de outros cursos e eles apresentaram características importantes como carga horária de mais de 30 horas/semanal, levando a sobrecarga o que, vem associado aos relatos de que durante a infância ou adolescência haviam passado por alguma situação que gerou algum tipo de sofrimento psíquico.

Graner e Cerqueira (2019) agruparam os fatores associados ao surgimento de TMCs em estudantes, e dividiu-os em seis categorias, a saber:

- Acadêmicas: dificuldade para conciliar os estudos, horas exaustivas de estudo, desconforto nas avaliações, insatisfação com o curso ou pouco interesse pelo mesmo;

- Saúde: ser tabagista, não realizar atividade física e vivenciar estresse;

- Sociodemográficas: sexo feminino, maior idade e baixa renda;

- Relacionais: dificuldade no relacionamento com os amigos, inadaptação à vida acadêmica, baixo apoio social.

- Psicológico: sentimentos negativos, não compartilhamento de problemas, baixa autoestima. 
- Social/Violência: ter sofrido discriminação, agressão e preocupação com a segurança pessoal.

Em contrapartida, Grether et al. (2019) observou uma prevalência na população estudada, formada por estudantes do curso de medicina, de 50,9\% de TMCs e classificou como grupo vulnerável. Sendo que, cerca de $60,6 \%$ nunca pensaram em abandonar o curso, mas demonstram ter uma carga horária extensa de oito a quatorze horas/dia e menos de duas horas de lazer por semana. Com relação a formação de novas amizades, eles não apresentaram dificuldades, nem houve rejeição por parte dos amigos e relataram receber apoio emocional adequado.

\section{CONCLUSÃO}

Por meio desta pesquisa foi possível abordar amplamente os Transtornos Mentais Comuns (TMCs) com foco nos estudantes universitários da área de saúde, sendo uma temática bastante relevante para os dias atuais; além disso foi possível evidenciar, através dos dados que os fatores causais associados ao surgimento dessas TMCs, indicados foram: a rotina de estudos excessiva, estresse, ausência de descanso e lazer, falta de apoio familiar e social.

Uma vez que as TMCs atuam sobre o desempenho acadêmico prejudicando a produtividade do estudante, sua qualidade de vida, bem-estar psicossocial, causando sofrimento mental e dificuldade de socialização dentro e fora da universidade. Portanto, esta pesquisa torna-se relevante pois instiga a comunidade estudantil ao autoconhecimento de seus sentimentos, medos, anseios e aos supostos gatilhos que os levam a entrar em estado de crise, além da conscientização quanto à procura por ajuda especializada para o melhor enfrentamento das situações e vivências abordadas.

Vale ressaltar também a importância deste estudo para a comunidade acadêmica , no âmbito institucional no tocante ao desenvolvimento e sensibilização do cuidado, respeito e acolhimento destes estudantes, para a promoção de ações de apoio que auxiliem os mesmos a desenvolver mecanismos que promovam saúde 
mental, emocional e física para lidar com as mais diversas vivências em sua caminhada acadêmica.

\section{REFERÊNCIAS BIBLIOGRÁFICAS}

ANACLETO-ESTRELA, Yoshyara da Costa et al. Estresse e correlatos com características de saúde e sociodemográficas de estudantes de medicina. Ces Medicina, [s.I.], v. 32, n. 3, p.215225, dez. 2018.

ARIÑO, Daniela Ornellas; BARDAGI, Marúcia Patta. Relação entre Fatores Acadêmicos e a Saúde Mental de Estudantes Universitários. Revista Psicologia em Pesquisa, Juiz de Fora, v. 12, n. 3, p.44-52, 28 dez. 2018.

AUERBACH, R. P. et al. Mental disorders among college students in the World Health Organization World Mental Health Surveys. Psychological Medicine, [s.l.], v. 46, n. 14, p.29552970, 3 ago. 2016.

COSTA, Kercia Mirely et al. Ansiedade em universitários na área da saúde. In: II Congresso Brasileiro das Ciências da saúde. 2017.

COSTA, Edméa Fontes de Oliva et al. Sintomas depressivos entre internos de medicina em uma universidade pública brasileira. Revista da Associação Médica Brasileira, Salvador, v. 58, n. 1, p.53-59, jan. 2012.

FACUNDES, Vera Lúcia Dutra; LUDERMIR, Ana Bernarda. Common mental disorders among health care students. Brazilian Journal of Psychiatry, v. 27, n. 3, p. 194-200, 2005.

FIOROTTI, Karoline Pedroti et al. Transtornos mentais comuns entre os estudantes do curso de medicina: prevalência e fatores associados. Jornal Brasileiro de Psiquiatria, Espírito Santo, v. 59, n. 1, p.17-23, 2010.

GRANER, Karen Mendes; CERQUEIRA, Ana Teresa de Abreu Ramos. Revisão integrativa: sofrimento psíquico em estudantes universitários e fatores associados. Ciência \& Saúde Coletiva, São Paulo, v. 24, n. 4, p.1327-1346, abr. 2019.

GRETHER, Eduardo Otávio et al. Prevalência de Transtornos Mentais Comuns entre Estudantes de Medicina da Universidade Regional de Blumenau (SC). Revista Brasileira de Educação Médica, Brasília, v. 43, n. 11, p.276-285, 2019.

LEÃO, Andrea Mendes et al. Prevalência e Fatores Associados à Depressão e Ansiedade entre Estudantes Universitários da Área da Saúde de um Grande Centro Urbano do Nordeste do Brasil. Revista Brasileira de Educação Médica, Brasília, v. 42, n. 4, p.55-65, dez. 2018.

LIMA, Sonia Oliveira et al. Prevalência da Depressão nos Acadêmicos da Área de Saúde. Psicologia: Ciência e Profissão, Brasília, v. 39, 2019.

NEPONUCENO, Hironaldo de Jesus; CARVALHO, Bárbara Dourado Nascimento de; NEVES, Nedy Maria Branco Cerqueira. Transtornos mentais comuns em estudantes de medicina. Revista Bioética, Salvador, v. 27, n. 3, p.465-470, set. 2019.

SANTOS, Lais Silva dos et al. QUALIDADE DE VIDA E TRANSTORNOS MENTAIS COMUNS EM ESTUDANTES DE MEDICINA. Cogitare Enfermagem, Bahia, v. 22, n. 4, 29 nov. 2017. 
SCHMIDT, Maria Inês et al. Chronic non-communicable diseases in Brazil: burden and current challenges. The Lancet, Rio Grande do Sul, v. 377, n. 9781, p.1949-1961, jun. 2011.

SERRA, Rosana Denobile; DINATO, Sandra Lopes Mattos e; CASEIRO, Marcos Montani. Prevalence of depressive and anxiety symptoms in medical students in the city of Santos. Jornal Brasileiro de Psiquiatria, Santos, v. 64, n. 3, p.213-220, 2015.

SILVA, Rodrigo Sinnott; DA COSTA, Letícia Almeida. Prevalência de transtornos mentais comuns entre estudantes universitários da área da saúde. Encontro: Revista de Psicologia, v. 15, n. 23, p. 105-112, 2015. 